\title{
Principe d'incertitude qualitatif pour les groupes de Lie nilpotents
}

\author{
Bouchta Boual and Mohammed HemdaOUI \\ Faculté des Sciences, \\ Département de Maths, \\ Université Mohammed Premier, \\ Oujda, Maroc. \\ bouali@sciences.univ-oujda.ac.ma \\ hemdaoui@sciences.univ-oujda.ac.ma
}

Recibido: 17 de Julio de 2001

Aceptado: 6 de Octubre de 2003

\section{ABSTRACT}

We prove that any simply connected nilpotent Lie group satisfies the qualitative uncertainty principle.

Key words: nilpotent Lie groups, representations, orbit method.

2000 Mathematics Subject Classification: 22E25, 22E27.

\section{Introduction}

Soit $\mathbb{R}^{n}$ muni de la mesure de Lebesgue $\lambda$ et soit $f$ une fonction $\lambda$-intégrable. Notons par :

$$
A_{f}=\left\{x \in \mathbb{R}^{n}: f(x) \neq 0\right\} \quad \text { et } \quad B_{f}=\left\{x \in \mathbb{R}^{n}: \hat{f}(x) \neq 0\right\},
$$

où $\hat{f}$ est la transformée de Fourier de $f$. Le but de ce travail est de généraliser le suivant résultat de Benedicks ([1]) :

Théorème 1.1. Si $f \in L^{1}\left(\mathbb{R}^{n}\right)$ vérifie :

$$
\lambda\left(A_{f}\right)<\infty \quad \text { et } \quad \lambda\left(B_{f}\right)<\infty,
$$

alors fest nulle $\lambda$-presque partout. 
Soit $G$ un groupe topologique, $m$ une mesure de Haar sur $G$ et $\mu$ la mesure de Plancherel associée à $m$.

Pour $f \in L^{1}(G)$ notons par :

$$
\begin{aligned}
& A_{f}=\{x \in G: f(x) \neq 0\} \\
& B_{f}=\{\pi \in \hat{G}: \hat{f}(\pi) \neq 0\}
\end{aligned}
$$

On dit que le groupe $G$ vérifie le principe d'incertitude qualitatif s' il n'existe pas de fonction $m$-intégrable non nulle vérifiant $m\left(A_{f}\right)<\infty$ et $\mu\left(B_{f}\right)<\infty$.

Dans ce papier on considère un groupe de Lie nilpotent connexe et simplement connexe $G$ et on établit qu'un tel groupe vérifie le principe d'incertitude qualitatif. Pour cela on considère $\mathcal{G}$ son algèbre de Lie et $\mathcal{G}^{*}$ le dual de $\mathcal{G}$ et on utilise l'application de Kirillov, qui identifie l'ensemble $\hat{G}$ des classes d'équivalence des représentations unitaires irréductibles de $G$ à l'ensemble des orbites de $\mathcal{G}^{*}$ sous l'action du groupe de Lie $G$.

\section{Notations et Généralités}

\subsection{Fonction module sur un groupe topologique}

Soit $G$ un groupe localement compact et $E$ un ensemble. Pour toute application

$$
f: G \rightarrow E
$$

et tout $s$ de $\mathrm{G}$, on notera par $\gamma(s) f$ et $\delta(s) f$ les applications de $G$ dans $E$ définies respectivement par :

$$
\gamma(s) f(x)=f\left(s^{-1} x\right) \quad \text { et } \quad \delta(s) f(x)=f(x s) .
$$

Translatées à gauche et à droite de $f$ par $s$, il en résulte que

$$
\gamma(s t) f=\gamma(s)(\gamma(t) f) \quad \text { et } \quad \delta(s t) f=\delta(s)(\delta(t) f) .
$$

Etant donnée une mesure de Haar $\mu$ sur $G$, on note par $\gamma(s t) \mu$ et $\delta(s) \mu$ les mesures sur $G$ images de $\mu$ par les homéomorphismes $x \mapsto s . x$ et $x \mapsto x s^{-1}, \gamma(s) \mu(f)=$ $\mu\left(\gamma\left(s^{-1}\right) f\right)$ et $\delta(s) \mu(f)=\mu\left(\delta\left(s^{-1}\right) f\right)$. On dit que la mesure est invariante à gauche (resp. à droite) si pour tout $s$ de $G$ on a

$$
\gamma(s) \mu=\mu(\operatorname{resp} . \delta(s) \mu=\mu) .
$$

Sur un groupe localement compact, il est clair que

$$
\gamma(t)(\delta(s) \mu=\delta(s)(\gamma(t) \mu)=\delta(s) \mu .
$$

Alors $\delta(s) \mu$ est une mesure invariante à gauche. Il existe un nombre unique noté $\Delta_{G}(s)>0$, tel que

$$
\delta(s) \mu=\Delta_{G}(s) \mu .
$$


Ce nombre est indépendant de la mesure choisie voir ([2, chap. 14]).

On dit que $s \mapsto \Delta_{G}(s)$ est la fonction module sur $G$. En particulier, si $A$ est $\mu$-integrable, As l'est aussi et $\mu(A s)=\Delta_{G}(s) \mu(A)$.

\subsection{Rappels sur les représentations des groupes de Lie nilpotents}

\subsubsection{Quelques GÉNÉRALITÉS}

Soit $G$ un groupe topologique localement compact et $\hat{G}$ son dual topologique (qui s'identifie à l'ensemble des représentations unitaires irréductibles de $G$ ). $\operatorname{Si} G$ est muni d'une mesure de Haar, alors

$$
\lambda: G \rightarrow L^{2}(G)
$$

donnée par

$$
\lambda(s)(f)=f\left(s^{-1} x\right), f \in L^{2}(G), x \in G
$$

est dans $\hat{G}$.

\subsubsection{Représentations induites.}

Soit $N$ un sous-groupe fermé de $G$, soit m (resp. $\left.m_{N}\right)$ une mesure de Haar sur $G$ (resp. $N$ ), soit $\rho$ une représentation de $N$ dans $H_{\rho}$; nous allons définir la représentation induite de $\rho$ sur $G$ à partir de $N$, notée $\operatorname{Ind}_{G}^{N}(\rho)$.

Soit $E_{0}$ l'espace des fonctions continues $\phi$ de $G$ dans $H_{\rho}$ vérifiant :

(i) $\phi(s h)=\rho(h)^{-1} \phi(s) s \in G, h \in N$,

(ii) $\operatorname{supp}(\phi)$ est compact modulo $N$.

Sur $E_{0}$, le groupe agit par translation à gauche. Soit $\nu$ une mesure quasi-invariante sur $G / H$ de poids $c$, cette mesure nous permet de définir une norme sur $E_{0}$, de la façon suivante :

$$
\|\phi\|_{p}^{p}=\int_{G / N}\|\phi(s)\|_{H_{\rho}}^{p} d \nu(s), \quad \phi \in E_{0} .
$$

Nous définissons des espaces de Banach $E_{p}$ qui contiennent $E_{0}$ (comme sous-espace dense) et sur lequels $G$ agit par des isométries.

Nous pouvons définir une action isométrique, $\pi_{p}, 1 \leq p<\infty$ de $G$ sur $\left(E_{0},\|\cdot\|\right)$ en posant

$$
\pi_{p}(s) \phi(x)=c(s, x)^{1 / p} \phi\left(s^{-1} x\right) \quad \forall x \in G / N, \forall s \in G
$$

Nous remarquons que $\pi_{p}$ est continue et isométrique, si nous désignons par $E_{p}$ le complété de $E_{0}$ pour la norme $\|\cdot\|_{p}$.

Nous obtenons une représentation isométrique $\pi_{p}$ de $G$ dans l'espace $E_{p}$. Si $p=2$, cette représentation sera notée $\pi_{2}=\operatorname{Ind}_{G}^{N}(\rho)$.

Remarque 2.1. Si $\rho$ est la représentation triviale sur $N$, alors $E_{0}$ est l'ensemble des fonctions continues constantes sur les classes suivant $N$. 


\subsection{Représentation adjointe d'un groupe de Lie}

Soit $G$ un groupe de Lie, $\mathcal{G}$ son algèbre de Lie et $\mathcal{G}^{*}$ l'espace dual de $\mathcal{G}$. Le groupe $G$ agit sur $\mathcal{G}$ par la représentation adjointe

$$
\operatorname{ad}(g): \mathcal{G} \longrightarrow \mathcal{G}
$$

où $\operatorname{ad}(g)$ est la différentielle de l'automorphisme intérieur $i_{g}\left(i_{g}(h)=g h g^{-1}\right)$ en l'élément unité de $G$ et agit sur $\mathcal{G}^{*}$ par la $K$-représentation ou représentation coadjointe $K$ :

$$
K(g): \mathcal{G}^{*} \longrightarrow \mathcal{G}^{*}, \quad\langle K(g) \cdot F, X\rangle=\left\langle F, a d\left(g^{-1}\right) \cdot X\right\rangle \quad \forall X \in \mathcal{G} .
$$

Notons par $O(G)$ l'ensemble des orbites du groupe de Lie dans la $K$-représentation, muni de la topologie quotient de la topologie naturelle dans $\mathcal{G}^{*}$.

On sait que toute orbite est munie d'une structure de variété symplectique homogène.

Soit $H$ un sous groupe normal (de codimension 1) de $G$, donc le groupe $G$ est un produit semi-direct de $H$ et d'un certain sous groupe $S$ isomorphe à $\mathbb{R}$ [3]. Nous désignons par la suite $\mu_{1}$ la mesure de Haar sur $H$ et par $\lambda$ celle de Lebesgue sur $\mathbb{R}$.

\section{Principe IQ pour les groupes de Lie nilpotents.}

Dans cette partie nous montrons que tous les groupes de Lie nilpotents vérifient le principe d'incertitude qualitatif. Pour ceci nous utilisons la relation reliant la mesure de Plancherel sur $G$ et celle sur son sous-groupe dérivé due à Garimella [4], et le résultat de désintégration des représentations due à Baklouti et Ludwig [8].

\subsection{Transformée de Fourier}

En utilisant [6], nous définissons la transformée de Fourier à valeurs opérateurs. Soit $F \in \mathcal{G}^{*}$, il existe une suite de sous-algèbres de Lie $\mathcal{G}_{j}$ de $\mathcal{G}$ telles que :

(i) $\operatorname{dim} \mathcal{G}_{j}=m-j$,

(ii) $\mathcal{G}=\mathcal{G}_{0} \supset \mathcal{G}_{1} \supset \cdots \supset \mathcal{G}_{m}=(0)$,

(iii) $\left[\mathcal{G}, \mathcal{G}_{j}\right] \subset \mathcal{G}_{j+1}$

où $\mathcal{G}_{1}$ est une polarisation en $F$ [7]. Soit $\chi_{F}$ le caractère défini sur $\mathcal{G}_{1}$ par

$$
\left\langle\chi_{F}, \exp (X)\right\rangle=e^{i\langle F, X\rangle} \quad \forall X \in \mathcal{G}_{1}
$$

On définit par induction une représentation $\pi_{F}$ de $G$ par

$$
\pi_{F}=\operatorname{Ind}_{G_{1}}^{G} \pi_{F_{1}} \quad \text { où } \quad \pi_{F_{1}} \in \hat{G}_{1} .
$$


Pour tout $f \in C_{c}^{\infty}(G)$ et $\pi_{F} \in \hat{G}$, on définit la transformée de Fourier opérateur par

$$
\hat{f}\left(\pi_{F}\right)=\int_{G} f(g) \pi_{F}(g) d g .
$$

Remarque 3.1. On pose $F^{s}=K(\exp (-s X)) \cdot F$, alors

$$
\pi_{F^{s}}(g)=\pi_{F}(\exp (s X) g \exp (-s X)) .
$$

Soient $F$ dans $\mathcal{G}$ et $F_{1}$ la restriction de $F$ à $\mathcal{G}_{1}$, notons par $\pi_{F_{1}}$ la représentation de $G_{1}$. Soit $\mu_{1}$ la mesure de Haar sur $G_{1}$, d'où on déduit il existe une mesure quasiinvariante sur $G / G_{1}$.

Soit $E$ l'espace de toutes les fonctions sur $G$ vérifiant :

(i) $\phi\left(g . g_{1}\right)=\pi\left(g_{1}\right) \phi(g)$,

(ii) $\int_{G / G_{1}}|\phi(s)| d \lambda(s)<\infty$.

La représentation induite, notée par $\pi_{F}=\operatorname{Ind}_{G_{1}}^{G} \pi_{F_{1}}$, est donnée par

$$
\pi_{F}(g) \phi(x)=\phi(g \cdot x) \quad \forall x \in G / G_{1}, \quad g \in G, \quad \phi \in E .
$$

Mais $E$ est isomorphe à $L^{2}(\mathbb{R} \cdot X) \approx L^{2}(\mathbb{R})$, où $X$ est un élément de $\mathcal{G} / \mathcal{G}_{1}$. Soit $\phi$ considérée comme élément de $L^{2}(\mathbb{R})$ et calculons $\hat{f}\left(\pi_{F}\right) \phi$ au point $\exp (s X)$, où $\phi \in L^{2}(\mathbb{R}):$

$$
\begin{aligned}
& \hat{f}\left(\pi_{F}\right) \phi(\exp (s X))=\int_{G} f(g) \pi_{F}(g) \phi(\exp (s X)) d g \\
&=\int_{G} f(g) \phi(\exp (s X \cdot g) d g \\
&\left.=\int_{\mathbb{R}} \int_{G_{1}} f(h \cdot \exp (t X)) \phi(\exp (s X) \cdot h \cdot \exp (t X)) d t d h\right) \\
&=\int_{\mathbb{R}} \int_{G_{1}} f(h \cdot \exp (t X)) \phi(\exp (s X) \cdot h \\
&\cdot \exp (-s X) \exp ((s+t) X)) d t d h .
\end{aligned}
$$

Posons $f^{t}(h)=f(h \cdot \exp (t X))$. D'après la remarque 3.1 on a

$$
\hat{f}\left(\pi_{F}\right) \phi(\exp (s X))=\int_{\mathbb{R}} \hat{f}^{t}\left(\pi_{F_{1}} s\right) \phi(\exp ((s+t) X) d t .
$$

Lemme 3.2. $f \in \operatorname{Ker}\left(\pi_{F}\right)$ si et seulement si $f^{t} \in \operatorname{Ker}\left(\pi_{F^{s_{1}}}\right)$ pour tout $s \in \mathbb{R}$ et pour presque tout $t \in \mathbb{R}$. 
Démonstration. Soit $\operatorname{Ker}\left(\pi_{F}\right)$ le noyau de la représentation $\pi_{F}$ de $G$. Si $f \in \operatorname{Ker}\left(\pi_{F}\right)$, et pour tout $\phi$ on a d'après la formule (1),

$$
\int_{\mathbb{R}} \hat{f}^{t}\left(\pi_{F^{s_{1}}}\right) \phi(\exp ((s+t) X)) d t=0
$$

ce qui implique que pour presque tout $t \in \mathbb{R}, \hat{f}^{t}\left(\pi_{F_{1}} s\right)=0, \forall s \in \mathbb{R}$ et $f^{t}$ est dans $\operatorname{Ker}\left(\pi_{F_{1}} s\right)$.

Réciproquement pour presque tout $t$ et tout $s$ dans $\mathbb{R}$, si $\hat{f}^{t} \in \operatorname{Ker}\left(\pi_{F^{s_{1}}}\right)$, alors $\hat{f}\left(\pi_{F}\right)=0$, ce qui montre que $f$ est dans le noyau de $\pi_{F}$.

Remarque 3.3. On peut traduire le lemme 3.2, en termes de $B_{f}$ et $B_{f^{t}}$ de la manière suivante :

$$
\pi_{F} \in B_{f} \Longleftrightarrow \pi_{F_{1} s} \in B_{f^{t}} \text { pour presque tout t et } \forall s \in \mathbb{R} \text {. }
$$

\subsection{Mesure de Plancherel sur le dual topologique}

Soit $G$ un groupe de Lie nilpotent, simplement connexe d'algèbre de Lie $\mathcal{G}, \mathcal{G}^{*}$ son dual. Soit $\mathcal{B}=\left\{X_{1}, \ldots, X_{n}\right\}$ une base de $\mathcal{G}$ et $\mathcal{B}^{*}=\left\{X_{1}^{*}, \ldots, X_{n}^{*}\right\}$ la base duale de $\mathcal{G}^{*}$. Soit $F \in \mathcal{G}^{*}$ de classe maximale, il lui correspond une orbite, sous l'action de $G, \mathcal{O}_{F}=\operatorname{Ad}^{*}(G) \cdot F$ de dimension maximale $2 k$. Soit $r=n-2 k$ et $2 k$ indices $\left\{i_{1}, \ldots, i_{2 k}\right\} \subseteq\{1, \ldots, n\}$ et les $r$ indices $\left\{d_{1}, \ldots, d_{r}\right\}$ qui restent. Considérons $\mathcal{B}_{d}^{*}=\left\{X_{d_{1}}^{*}, \ldots, X_{d_{r}}^{*}\right\}, W_{d}$ l'espace engendré par $\mathcal{B}_{d}^{*}$ qui correspond bijectivement à $\hat{G}$. Donc la mesure de Plancherel sur $\hat{G}$ est donnée, à l'aide de la mesure de Lebesgue $d m$ sur $W_{d}$, par

$$
d \mu=|\operatorname{Pf}(F)| d m,
$$

où $\operatorname{Pf}(F)$ est le Pfaffian de $F, F \in W_{d}$.

Soit une suite

$$
0=\mathcal{G}_{n} \subset \mathcal{G}_{n-1} \subset \cdots \subset \mathcal{G}_{1} \subset \mathcal{G}_{0}=\mathcal{G}
$$

d' idéaux de $\mathcal{G}$ telle que la dimension de $\mathcal{G}_{i}$ soit $i$ pour tout $i \in\{1, \ldots, n\}$. On note $\mathcal{G}^{F}$ le radical de $F$ dans $\mathcal{G}$.

Soit $G_{1}$ le sous-groupe analytique de $G$ d'algèbre de Lie $\mathcal{G}_{1}$. $G_{1}$ est un sous-groupe de codimension 1 de $G$, et utilisons la relation entre la mesure de Plancherel sur $G$ et celle sur $G_{1}$, due à G. Garimella [4, section 3]. On distingue deux cas :

$1^{\text {er }}$ cas : Si $\mathcal{G}^{F} \subset \mathcal{G}_{1} \quad \forall F \in W_{d}, d \mu_{1}=q(F) d \mu d F_{j_{1}}$.

$2^{\text {ème }}$ cas : $\operatorname{Si} \mathcal{G}^{F} \not \subset \mathcal{G}_{1} d \mu=d \mu_{1} \times d F_{1}$.

\subsection{Théorème}

Avant d'énoncer le résultat principal de ce papier, nous donnons un lemme. Soit $f$ une fonction $\mu$-intégrable, soit $G_{1}$ un sous-groupe normal de $G$ muni d'une mesure 
$\mu_{1}$. Alors, il existe une mesure quasi-invariante $\nu$ sur $G / G_{1}$ telle que

$$
\int_{G} f(g) d \mu(g)=\int_{G / G_{1}} \int_{G_{1}} f(g n) d \mu_{1}(n) d \nu(g) .
$$

Notons par :

$$
A_{f^{g}}=\left\{n \in G_{1}: f^{g}(n) \neq 0\right\}
$$

Lemme 3.4. Si $\mu\left(A_{f}\right)$ est fini, alors $\mu_{1}\left(A_{f^{g}}\right)$ est fini pour presque tout $g \in G / G_{1}$.

Démonstration. Calculons $\mu\left(A_{f}\right)$. Comme

$$
\begin{aligned}
\mu\left(A_{f}\right) & =\int_{G} 1_{A_{f}}(g) d \mu(g) \\
& =\int_{G / G_{1}} \int_{G_{1}} 1_{A_{f^{g}}}(n) d \mu_{1}(n) d \nu(g) \\
& =\int_{G / G_{1}} \mu_{1}\left(A_{f^{g}}\right) d \nu(g)<\infty,
\end{aligned}
$$

alors $\mu_{1}\left(A_{f^{g}}\right)$ est fini $\mu_{1}$-presque partout et pour presque tout $g \in G / G_{1}$.

Théorème 3.5. Tout groupe de Lie G simplement connexe et nilpotent vérifie le principe d'incertitude qualitatif.

Démonstration. Raisonnons par induction sur la dimension $p$ de $\mathrm{G}$.

Si $p=1$, alors $G$ est isomorphe à $\mathbb{R}$ et le résultat est vrai.

Soit $G$ un groupe de dimension $p$ et supposons que l'affirmation soit pur tout groupe de dimension $k<p$. Soit $F$ un élément du dual $\mathcal{G}^{*}$ de $\mathcal{G}$ et soit $\mathcal{H}$ une sous algèbre de $\mathcal{G}$ subordonnée à $F$. Soit $\mathcal{B}=\left\{X_{1}, \ldots, X_{r}\right\}$ une base de Malcev relative à $\mathcal{H}$. Le sous espace $\mathcal{G}_{1}$ engendré par $\mathcal{H}, X_{1}, \ldots, X_{r-1}$ est un idéal de codimension 1 de $\mathcal{G}$ et $\left\{X_{1}, \ldots, X_{r-1}\right\}$ est une base de Malcev de $\mathcal{G}_{1}$ relative à $\mathcal{H}$. Donc $G_{1}$, le sous-groupe analytique de $G$, d'algèbre de Lie $\mathcal{G}_{1}$, est un sous-groupe de Lie nilpotent connexe et simplement connexe. Supposons que $m\left(A_{f}\right)$ et $\mu\left(B_{f}\right)$ soient finis. D'après le lemme 3.4, pour presque tout $t, m_{1}\left(A_{f^{t}}\right)$ est fini. Pour conclure, il reste à montrer que $\mu_{1}\left(B_{f^{t}}\right)$ est fini. Pour ceci on distingue deux cas :

(i) Les G-orbites sont saturées par rapport à $\mathcal{G}_{1}{ }^{\perp}$, donc pour presque tout $\phi$ de $\mathcal{G}^{*}$. Soit $\phi_{0}$ restriction de $\phi$ à $\mathcal{G}_{1}^{*}, \pi_{\phi}=\operatorname{Ind}_{G_{1}}^{G} \pi_{\phi_{0}}$ est irréductible. D'après [8, proposition 2.5] on a

$$
\int_{\mathcal{G}_{1}^{*}}^{\oplus} \operatorname{Ind}_{G_{1}}^{G} \pi_{\phi_{0}} d \lambda_{\mathcal{G}_{1}}\left(\phi_{0}\right) \simeq \int_{\mathcal{G}^{*}}^{\oplus} \pi_{\phi} d \lambda_{\mathcal{G}^{*}}(\phi)
$$

où $\lambda_{\mathcal{G}^{*}}$ est la mesure de Lebesgue sur $\mathcal{G}^{*}$ et $\lambda_{\mathcal{G}_{1}^{*}}$ est celle sur $\mathcal{G}_{1}^{*}$ 
De cette formule et vu la forme de $X_{r}$ on conclut que l'application

$$
\phi \longrightarrow \phi_{0}
$$

est un isomorphisme respectant les mesures $\lambda_{\mathcal{G}_{1}{ }^{*}}$ et $\lambda_{\mathcal{G}^{*}}$, donc

$$
\mu_{1}\left(B_{f^{t}}\right)=\mu\left(B_{f}\right)<\infty
$$

Appliquons l'hypothèse de induction; $f^{t}$ est nulle $m_{1}$-presque partout, et par suite $f$ est nulle $m$-presque partout sur $G$.

(ii) Les $G$-orbites ne sont pas saturées par rapport à $\mathcal{G}_{1}{ }^{\perp}$. Choisissons pour chaque $\phi_{0}$ de $\mathcal{G}_{1}{ }^{*}$ l'extension $\phi$ définie par $\phi\left(B_{r}\right)=0$. Ceci nous donne

$$
i n d_{G_{1}}^{G} \pi_{\phi_{0}} \simeq \int_{\mathbb{R}}^{\oplus} \pi_{\phi_{0}+s B_{r}^{*}} d s,
$$

d'où $\mu\left(B_{f}\right)=\int_{\mathbb{R}} \mu_{1}\left(B_{f^{t}}\right) d t<\infty$.

Donc pour presque tout $t \in \mathbb{R}, \mu_{1}\left(B_{f^{t}}\right)$ est finie. D'après l'hypothèse de récurrence pour presque tout $t \in \mathbb{R}$, on a $f^{t}=0$. Donc $f$ est nulle pour presque tout $h \in G_{1}$ et pour presque tout $t \in \mathbb{R}$, ce qui se traduit par $f$ est nulle $m$-presque partout sur G.

Remarque 3.6. Le théorème n'est pas vrai si on remplace $L^{1}(G)$ par l'espace des distributions tempérées sur $G$.

En effet, soit $T$ la distribution sur $G$ définie par :

$$
T=\Sigma_{\gamma \in \Gamma} \delta_{\gamma},
$$

où $\Gamma$ est un sous-groupe discret central de $G$, et $\delta_{\gamma}$ est la distribution de Dirac en $\gamma$. En appliquant la formule de Poisson [5], on obtient :

$$
\hat{T}=\Sigma_{\gamma \in \Gamma} \delta_{2 \pi \gamma} .
$$

Nous remarquons que $A_{T}$ et $B_{T}$ ont des mesures finies nulles, pourtant la distribution $T$ est non nulle.

\section{Références}

[1] M. Benedicks, On Fourier transforms of functions supported on sets of finite Lebesgue measure, J. Math. Anal. Appl. 106 (1985), 180-183.

[2] J. Dieudonné, Éléments d'analyse. Tome II : Chapitres XII à XV, Cahiers Scientifiques, Fasc. XXXI, Gauthier-Villars, Éditeur, Paris, 1968.

[3] A. Kirillov, Éléments de la théorie des représentations, Éditions Mir, Moscow, 1974. 
[4] G. Garimella, Un théorème de Paley-Wiener pour les groupes de Lie nilpotents, J. Lie Theory 5 (1995), 165-172.

[5] M. Rais, Représentation des groupes de Lie nilpotents et méthode des orbites, Nancy, 1980, Cours du C.I.M.P.A., Analyse harmonique.

[6] L. Baggett and A. Kleppner, Multiplier representations of abelian groups, J. Functional Analysis 14 (1973), 299-324.

[7] J. Ludwig and H. Zahir, Surjectivité de la transformation de Fourier adaptée nilpotente, Université de Metz U.A. C.N.R.S. 339 02/93.

[8] A. Baklouti and J. Ludwig, Désintégration des représentations monomiales des groupes de Lie nilpotents, J. Lie Theory 9 (1999), 157-191. 